\title{
Effect of Lossy Earth on Antenna Gain
}

\author{
Richard J. Coe and Walter L. Curtis \\ Contribution from Aero-Space Division, The Boeing Company, Seattle, Wash.
}

(Received March 6, 1963; revised September 23, 1963)

\begin{abstract}
The effect of lossy earth on the far field gain of antennas with finite size metal ground planes has been investigated experimentally. Gain measurements were made of several antennas and their associated ground planes mounted both flush with the surface of the earth and far removed from the earth. It was found that for the ground plane sizes used in this study the gains for the two situations differed only at low elevation angles. The results suggest that one may estimate the behavior of an antenna over conducting earth by measuring the free space gain utilizing simple modeling techniques on a conventional antenna pattern range.
\end{abstract}

\section{Introduction}

The radiation characteristics at small elevation angles are important in air-to-ground communication and troposcatter applications. This paper considers such radiation for low silhouette antennas which are mounted flush with the surface of the earth. Related to this is the problem, originally formulated by Sommerfeld, of determining the fields of elemental electric and magnetic dipoles located in the vicinity of a conducting half-space such as earth. The solution of the latter problem is the Green's function for this geometry; and once the current distribution is known, the far field may be calculated by an integration over the source currents. In this manner solutions have been obtained to the problem of linear wire antennas located in the region above a conducting half-space. An excellent summary of Sommerfeld's work, and that of subsequent contributors to the problem, may be found in R. W. P. King's, "The Theory of Linear Antennas" [1956].

In practice, antennas are constructed on a metallic ground plane of finite size in order to improve the coupling to the groundwave or skywave. Of particular importance is the effect of the earth on the far field pattern of the antenna. For this reason, the radiation patterns of several different antennas mounted on finite size metallic ground planes wera measured in the presence of the earth. The primary effect on the radiation pattern was found to occur at low elevation angles.

Theoretical considerations of simple antennas will give some insight into the problem. Consider for example a quarter-wavelength stub antenna mounted on a metallic ground plane which is several wavelengths in size. The current distribution in the vertical stub will essentially be unperturbed by the presence of the earth. An approximate solution for this problem using the concept of an impedance boundary at the earth-air interface has been presented by Wait [1963] and numerical solutions by
Wait and Walters [1962]. Their expression for the skywave component of the field is

$$
E \approx C\left(1+R_{v}\right)(1+\Omega) \frac{\cos (\pi / 2 \sin \psi)}{\cos \psi}
$$

where

$$
\begin{aligned}
C & =\text { constant of proportionality } \\
R_{v} & =\text { reflection factor for vertical polarization } \\
& =\frac{\epsilon_{r}(1-j \tan \delta) \sin \psi-\sqrt{\epsilon_{r}(1-j \tan \delta)-\cos ^{2} \psi}}{\epsilon_{r}(1-j \tan \delta) \sin \psi+\sqrt{\epsilon_{r}(1-j \tan \delta)-\cos ^{2} \psi}}
\end{aligned}
$$

$\psi=$ elevation angle

$\boldsymbol{\epsilon}_{r}=$ relative dielectric constant of earth

$\tan \delta=$ loss tangent of earth

$(1+\Omega)=$ correction factor computed by Wait and Walters

$$
\frac{\cos (\pi / 2 \sin \psi)}{\cos \psi}=\text { pattern factor for } \lambda / 4 \text { stub. }
$$

The measured pattern of a quarter wavelength stub is compared in figure 1 with the theoretical expression as given by (1).

The greatest variation with angle occurs from the factor $\left(1+R_{v}\right)$ which is a consequence of geometric optics for a plane interface when the ground plane effect is ignored. The same factor is obtained in the geometric optics approximation [King, 1956] for horizontal magnetic dipoles. Thus, we might expect low angle radiation from a slot in a finite size ground plane of moderate size to have approximately the same variation. This is confirmed by measurements taken on a half-wave slot, the results of which are shown in figure 1.

\section{Measurement Technique}

Gain was measured by probing the field about a given antenna with a calibrated or standard horn. The gain was determined from Schelkunoff and 


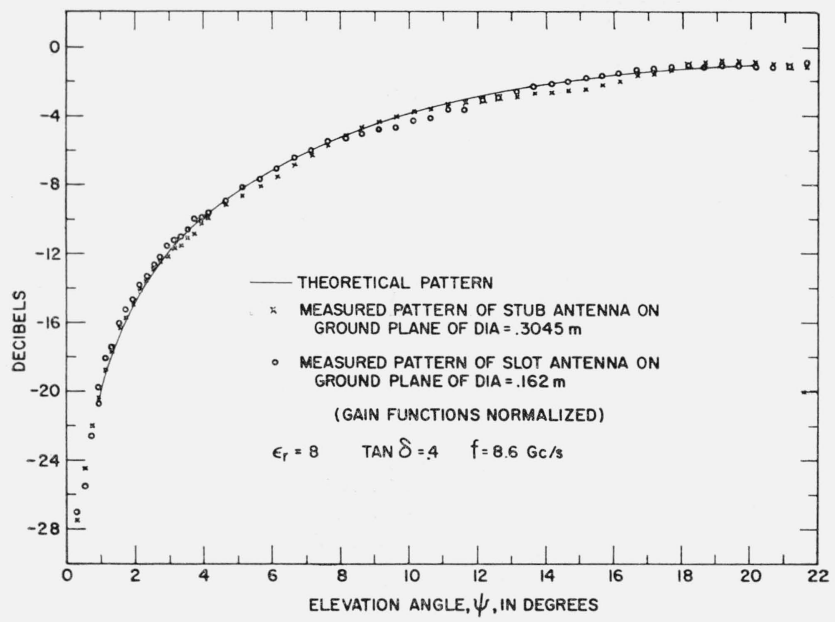

Figure 1. Comparison of measured and theoretical pattern of slot and stub antennas.

Friis [1952] transmission formula which can be written as

$$
G_{T}=\frac{16 \pi^{2} R^{2} P_{R}}{\lambda^{2} G_{R} P_{T}}
$$

where

$$
\begin{aligned}
G_{T} & =\text { gain of transmitting antenna } \\
& =\frac{\text { Far field radiation intensity (sky wave) }}{\text { Power input to antenna } / 4 \pi} \\
G_{R} & =\text { gain of receiving antenna } \\
P_{T} & =\text { transmitted power } \\
P_{R} & =\text { received power } \\
R & =\text { distance between antennas } \\
\lambda & =\text { wavelength. }
\end{aligned}
$$

A block diagram and a photograph of the equipment at the test site are shown in figures 2 and 3 respectively. The gain of the horn was computed using tables by Braun [1956] to be $19.17 \mathrm{db}$ at a frequency of $8.6 \mathrm{Gc} / \mathrm{s}$. The frequency of $8.6 \mathrm{Gc} / \mathrm{s}$ was used for all experimental measurements. The value of the radius $(R)$ shown in figure 2 was $5.67 \mathrm{~m}$. This requires, according to Braun [1953], a gain correction due to the near field of $-0.12 \mathrm{db}$. The net value of gain for the standard horn was thus $19.05 \mathrm{db}$.

The ratio of power received to power transmitted was found by initially connecting the detector and VSWR indicator to the source as shown by the dotted lines in figure 2 and then adjusting the gain of the VSWR indicator to read one. Thus, when connected to the receiving horn, the VSWR meter indicates directly the square root of the ratio of power in the directional coupler to power received. If $D$ is used to represent the reading of the VSWR meter then

$$
D=\frac{\sqrt{c}\left|V_{1}^{+}\right|}{\sqrt{P_{R}}}
$$

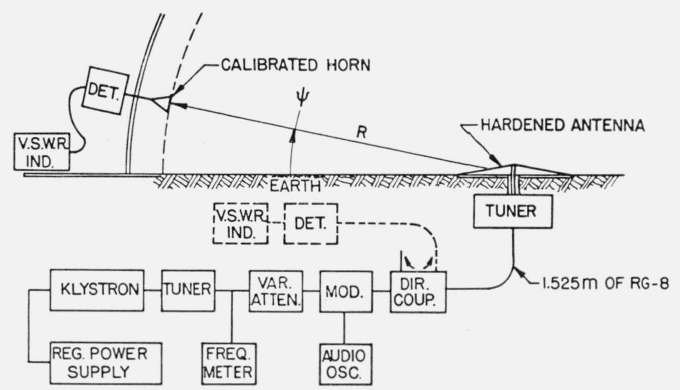

Figure 2. Block diagram of equipment used for antenna gain measurements.

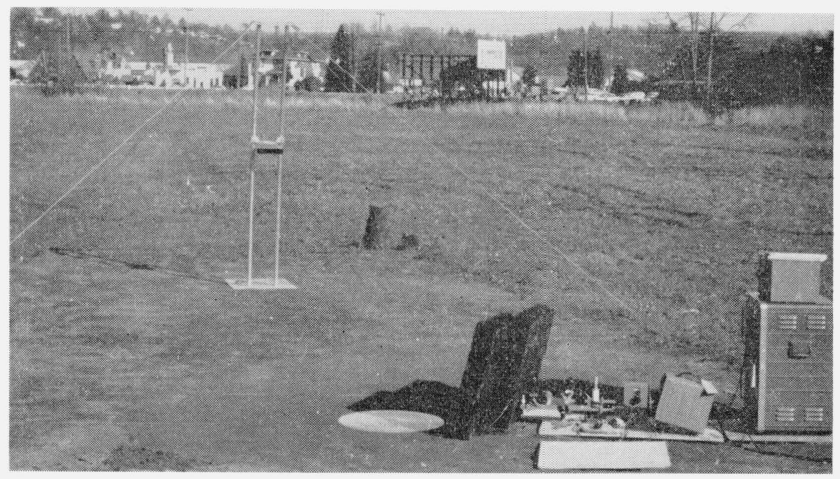

FiguRe 3. Equipment for measuring antenna gain.

where $V_{1}^{+}$represents the electric field incident from the generator and $c$ is the coupling factor of the directional coupler.

In order to avoid operating the detector over too large a dynamic range an accurately calibrated variable attenuator was used in series with the rf source. The attenuator was adjusted to maintain the power level at the detector within $3 \mathrm{db}$.

The equipment was given at least $30 \mathrm{~min}$ for warmup time which resulted in a calibration drift of less than $0.2 \mathrm{db}$ during the gain measurements of any one antenna.

It was necessary to use a short length of coaxial cable between the directional coupler and the antenna. For all measurements the tuner was adjusted to match the antenna to the coaxial line. Therefore, the transmitted power and incident voltage are related to the transmission coefficient $\left(S_{21}\right)$ of the coaxial line by

$$
\frac{\sqrt{P_{T}}}{\left|V_{1}^{+}\right|}=\left|S_{21}\right|
$$

Using (4) and (5), (3) becomes

$$
G_{T}=\frac{16 \pi^{2} R^{2} c}{\lambda^{2} G_{R} D^{2}\left|S_{21}\right|^{2}} .
$$

The value of $\left(S_{21}\right)$ was measured using the method of Deschamps [1953] and found to be 0.737 . The directional coupler used has a value of $c$ equal to $19.8 \mathrm{db}$. 
Using the above numerical values and converting to decibels results in the following final value for antenna gain.

$$
10 \log _{10} G_{T}=29.8-10 \log _{10} D .
$$

In order to check the above formula, a preliminary run was made on an antenna of known gain, namely, a half-wavelength slot. The slot was placed in the center of a circular metallic ground plane $0.314 \mathrm{~m}$ in diameter. For practical reasons the slot was milled in a $0.152 \mathrm{~m}$ diameter disk of brass with a larger disk of aluminum used to extend the ground plane. The plot in figure 4 shows the measured gain in decibels for the antenna mounted flush with the ground and the gain of the antenna and ground plane in free space. In the region above $15 \mathrm{deg}$, the correlation between the free space gain and the gain as measured over the earth is within $0.5 \mathrm{db}$, showing the accuracy of the experimental procedure.

As shown in figure 4 , the field is attenuated rapidly as $\psi$ approaches zero. This means that the receiving horn is illuminated nonuniformly by the test antenna causing an error in measured gain or equivalently an error in effective elevation angle. In order that this error be negligible a $0.0508 \mathrm{~m}$ vertical aperture was used.

\section{Description of Antennas}

In addition to the slot antenna mentioned in the preceding section, measurements were made on a quarter-wavelength stub located at the center of a circular metal disk. The stub was formed by extending the center conductor of a coaxial panel connector which was attached to the disk.

Two types of omnidirectional dielectric surface wave antennas were constructed for this study. One, having a major lobe in free space at approximately $13 \mathrm{deg}$ from the horizon, utilizes a flat metal surface covered with dielectric and the other with a major lobe at 8 deg utilizes a conical metal ground plane covered with dielectric. In each case the dielectric is a $0.305 \mathrm{~m}$ diameter polystyrene disk which is $0.635 \mathrm{~cm}$ thick at the center tapering linearly to zero thickness at the outer periphery. A sketch showing a cross section through the axis of symmetry of these antennas is shown in figure 5. The feed for these antennas is similar to that used with the stub antenna. Additional information on conical surface wave antennas may be found in an article by Jones and Folson [1953]. Measurements were also made on a 12 element end fire array utilizing parasitically coupled slots [Coe et al., 1960]. A photograph of this antenna mounted flush with the earth is shown in figure 6 . Provisions were made for changing the size of the finite metallic ground planes on all of the antennas used in the study.

\section{Results}

The results presented here were insensitive to small variations in the dielectric properties of the

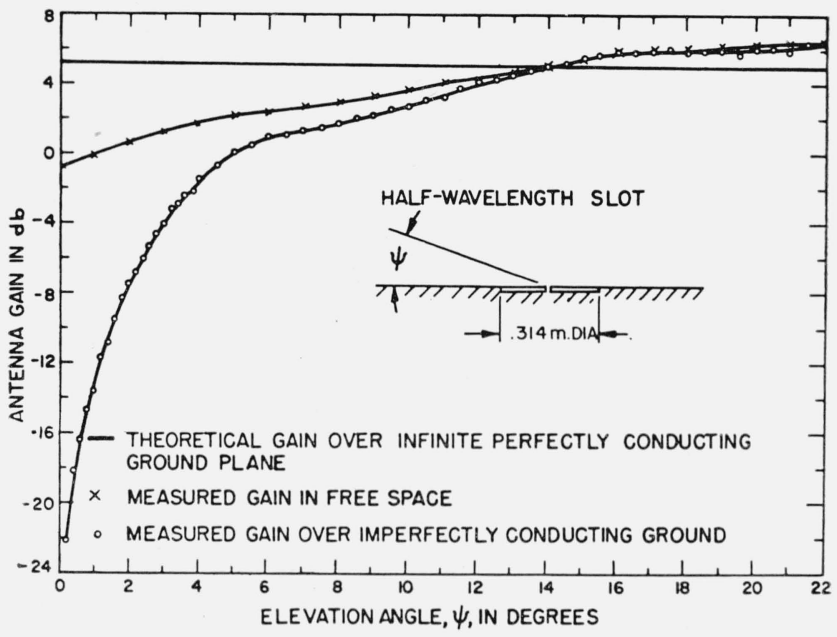

Figure 4. Measured gain of half-wavelength slot antenna mounted on a $0.314 \mathrm{~m}$ diameter ground plane.

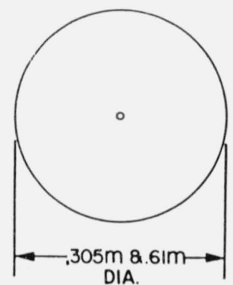

DIA

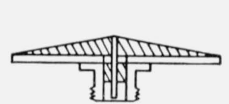

Figure 5. Sketch of dielectric surface wave antennas used in this study.

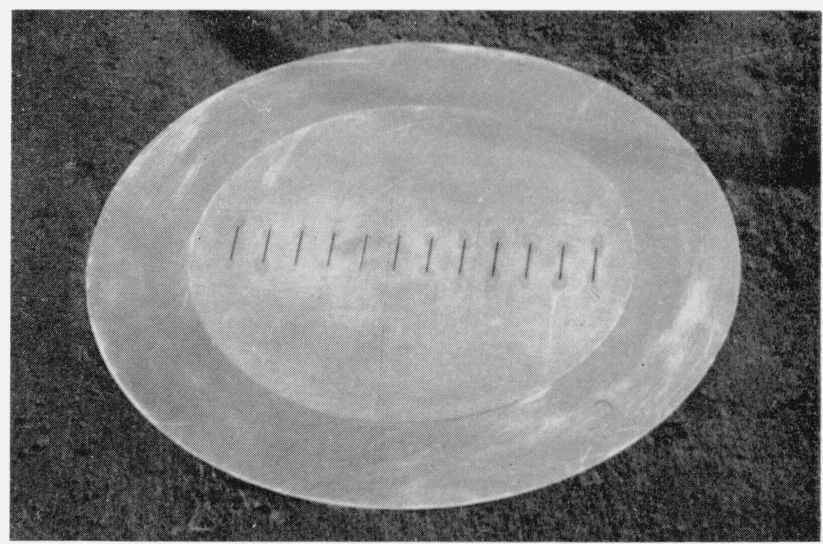

Figure 6. Parasitic slot array mounted on a flat metal ground plane. 


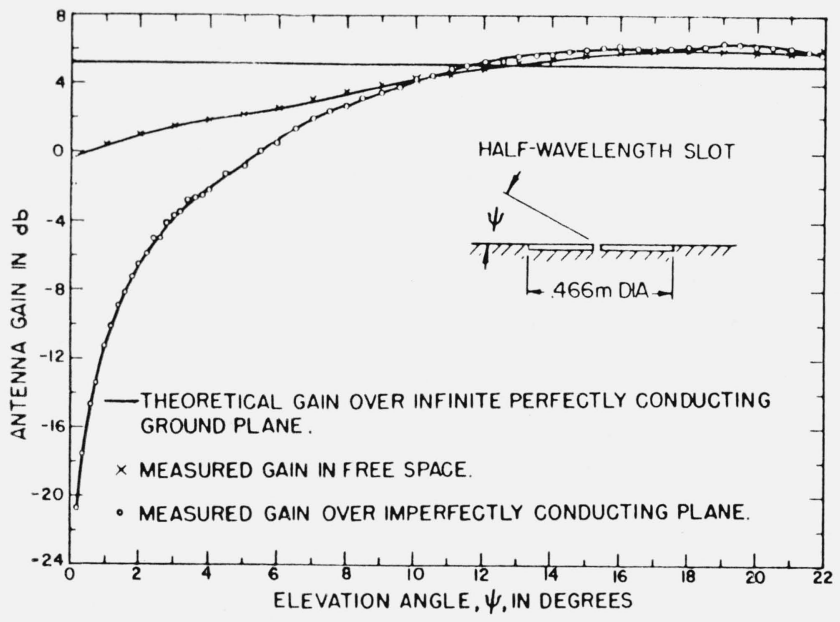

Figure 7. Measured gain of half-wavelength slot antenna mounted on a $0.466 \mathrm{~m}$ diameter ground plane.

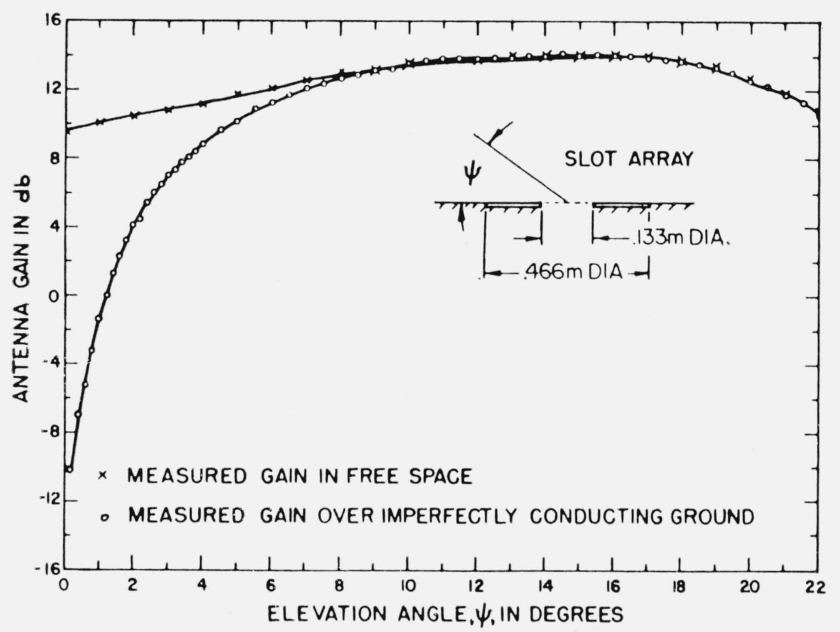

Figure 8. Measured gain of parasitic slot array mounted on a $0.466 \mathrm{~m}$ diameter ground plane.

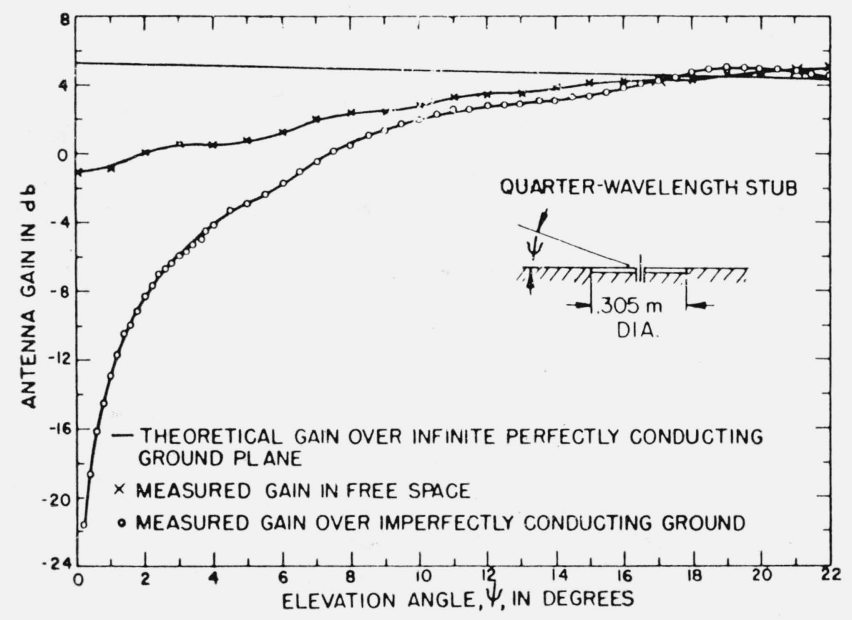

Figure 9. Measured gain of quarter-wavelength stub antenna mounted on a $0.305 \mathrm{~m}$ diameter ground plane.

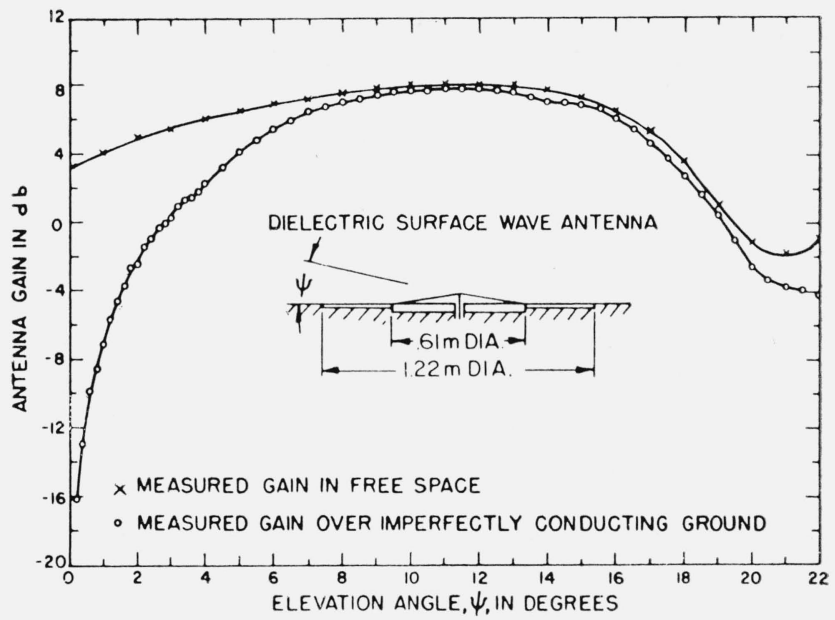

Figure 10. Measured gain of dielectric surface wave antenna mounted on a 1.22 $m$ diameter ground plane.

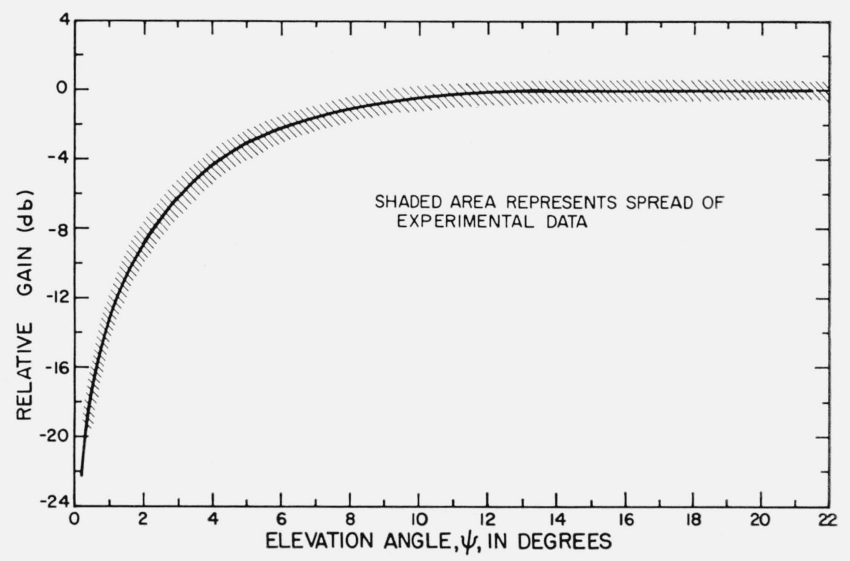

Figure 11. Average difference in db between free space gain and gain over earth for all antennas measured in this study.

earth observed concurrently with the measurements. The average values of relative dielectric constant and loss tangent were 9 and 0.5 , respectively. However, with different soils and at other frequencies the dielectric properties may vary appreciably.

Data was taken on each of the antennas with several sizes of ground plane ranging from approximately $0.23 \mathrm{~m}$ to $1.22 \mathrm{~m}$ in diameter. Typical data is shown in figures 7 through 10 . Shown in each figure are two curves plotted in rectangular coordinates of antenna gain in decibels with respect to the isotropic level for low elevation angles. One of the two curves represents the free space gain as measured on a pattern range while the other curve represents the data obtained with the antenna in the presence of the earth using the measurement technique described in this paper. It will be observed that in each the gain with and without the influence of the earth are nearly identical above an elevation angle of around $12 \mathrm{deg}$. As the elevation angle decreases to zero the antenna gain in each case 
decreases to zero in a manner similar to that indicated for the elemental dipoles. Data similar to that presented here was obtained on approximately seventeen different antenna and ground plane combinations. For comparison, the ratio between the free space gain and gain in the presence of the earth was plotted on a single graph for all the configurations measured. ${ }^{1}$ This leads to the conclusion that the ratio between the free space gain and gain on the earth was practically the same for all configurations. The average of this ratio is shown in figure 11 . This result suggests that one may estimate the behavior of an antenna over conducting earth by measuring the free space gain utilizing simple modeling techniques on a conventional pattern range. The gain over the ground may then be obtained by subtracting the curve of figure 11 from the measured free space gain.

1 A theoretical expression for the ratio of the free space gain and the gain on the earth has been derived using the correction factor $(1+\Omega)$ and will be published in a future paper.

\section{References}

Braun, E. H. (Jan. 1953), Gain of electromagnetic horns, Proc. IRE 41, No. 1, 109-115.

Braun, E. H. (Jan. 1956), Some data for the design of electromagnetic horns, IRE Trans. PGAP AP-4, No. 1, 29-31.

Coe, Richard J., G. Geld, and A. Ishimaru (Mar. 1960), A parasitic slot array, Tech. Rpt. No. 43, Department of Electrical Engineering, University of Washington.

Deschamps, G. A. (Aug. 1953), Determination of the reflection coefficients and insertion loss of a waveguide junction, J. Appl. Phys. 24, No. 8, 1046-1050.

Jones, E. M. T., and R. A. Folsom, Jr. (June 1953), A note on the circular dielectric disk antenna, Proc. IRE 41, 798.

King, R. W. P. (1956), Theory of linear antennas, p. 794 (Harvard University Press, Cambridge, Mass.).

Schelkunoff, S. A., H. T. Friis (1952), Antennas theory and practice, p. 185 (John Wiley \& Sons, Inc., New York, N.Y.).

Wait, J. R. (1963), The theory of an antenna over an inhomogeneous ground plane, preprinted from Electromagnetic Theory and Antennas, ed. by E. C. Jordon (Pergamon Press, Oxford).

Wait, J. R., and L. C. Walters (1963), Influence of a sector ground screen on the field of a vertical antenna, NBS Mono. No. 60.

(Paper 68D2-339) 